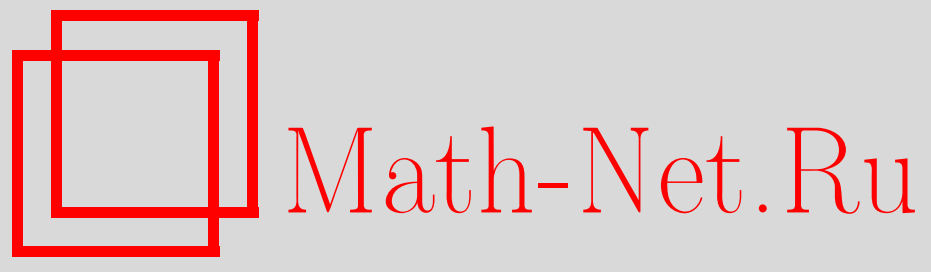

М. Э. Муминов, О конечности дискретного спектра оператора Шредингера трех частиц на решетке, $T M \Phi, 2008$, том 154, номер 2, 363-371

DOI: https://doi.org/10.4213/tmf6175

Использование Общероссийского математического портала Math-Net.Ru подразумевает, что вы прочитали и согласны с пользовательским соглашением http://www . mathnet.ru/rus/agreement

Параметры загрузки:

IP : 3.80 .181 .102

26 апреля 2023 г., 10:31:09

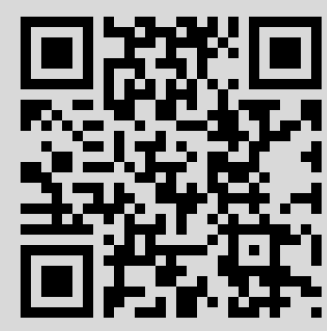




\title{
О КОНЕЧНОСТИ ДИСКРЕТНОГО СПЕКТРА ОПЕРАТОРА ШРЕДИНГЕРА ТРЕХ ЧАСТИЦ НА РЕШЕТКЕ
}

\begin{abstract}
Рассматривается система трех взаимодействующих с помощью парных короткодействующих потенциалов притяжения квантовых частиц (с бесконечной массой одной частицы) на трехмерной решетке. Доказана конечность числа связанных состояний соответствующего оператора Шредингера в случае, когда потенциалы удовлетворяют некоторым условиям и два двухчастичных подгамильтониана с бесконечными массами имеют резонанс в нуле, а для двухчастичного подгамильтониана с конечными массами нуль является регулярной точкой.
\end{abstract}

Ключевые слова: резонанс, двухчастичные подгамильтонианы, дискретный спектр, принцип вариации.

\section{1. ВВЕДЕНИЕ}

Существование бесконечного числа трехчастичных связанных состояний с отрицательной энергией трехчастичного непрерывного оператора Шредингера является одним из замечательных свойств спектрального анализа. Этот свойство впервые было обнаружено Ефимовым [1] и называется эффектом Ефимова. В работах [2], [3] существование бесконечного числа собственных значений трехчастичного оператора Шредингера было доказано в том случае, когда два из трех двухчастичных гамильтонианов имеют резонанс в нуле и нуль является регулярной точкой для третьего гамильтониана с конечной массой. В этих работах также обсуждался вопрос существования бесконечного числа собственных значений в случае, когда масса одной частицы обращается в бесконечность. Однако в этом случае вопрос о числе собственных значений оставался открытым, хотя в работе [4] высказано предположение о том, что число собственных значений $H$ конечно.

В настоящей работе рассматривается система трех квантовых частиц с бесконечной массой одной частицы на трехмерной решетке, взаимодействующих с помощью парных короткодействующих потенциалов притяжения. Для конкретного

${ }^{*}$ Самаркандский государственный университет им. Алишера Навои, Самарканд, Узбекистан. E-mail: mmuminov@mail.ru 
класса потенциалов доказана конечность числа связанных состояний соответствующего трехчастичного оператора Шредингера $H$ рассматриваемой системы на решетке в случае, когда два двухчастичных подгамильтониана с бесконечными массами имеют резонанс в нуле, а для подгамильтониана с конечными массами нуль является регулярной точкой. Таким образом, для конкретного класса потенциалов трехчастичного оператора Шредингера на решетке доказываются предположения, высказанные в работе [4].

\section{2. ОПИСАНИЕ ТРЕХЧАСТИЧНОГО ОПЕРАТОРА И ФОРМУЛИРОВКА ОСНОВНЫХ РЕЗУЛЬТАТОВ}

Пусть $\mathbb{Z}^{3}$ - трехмерная целочисленная решетка и $\ell_{2}\left(\left(\mathbb{Z}^{3}\right)^{3}\right)$ - гильбертово пространство квадратично-суммируемых функций, определенных на $\left(\mathbb{Z}^{3}\right)^{3}$.

Свободный гамильтониан $\widehat{H}_{0}$ системы трех произвольных квантовых частиц на решетке $\mathbb{Z}^{3}$ определяется как ограниченный самосопряженный оператор в гильбертовом пространстве $\ell_{2}\left(\left(Z^{3}\right)^{3}\right)$ :

$$
\widehat{H}_{0}=\frac{1}{2 m_{1}} \Delta_{x_{1}}+\frac{1}{2 m_{2}} \Delta_{x_{2}}+\frac{1}{2 m_{3}} \Delta_{x_{3}},
$$

где $\Delta_{x_{1}}=\Delta \otimes I \otimes I, \Delta_{x_{2}}=I \otimes \Delta \otimes I$ и $\Delta_{x_{3}}=I \otimes I \otimes \Delta ; m_{\alpha}>0$ - масса частицы $\alpha=\overline{1,3} ; \Delta$ - решетчатый лапласиан - есть разностный оператор, описывающий перенос частицы с узла на соседний узел, т.е.

$$
(\Delta \hat{\psi})(x)=\sum_{|s|=1}[\hat{\psi}(x)-\hat{\psi}(x+s)], \quad \hat{\psi} \in \ell_{2}\left(\mathbb{Z}^{3}\right) .
$$

Трехчастичный гамильтониан $\widehat{H}$ системы трех квантовых частиц с парными короткодействующими потенциалами $\hat{v}_{i j}, 1 \leqslant i<j \leqslant 3$, определяется как ограниченное возмущение свободного гамильтониана $\widehat{H}_{0}$ :

$$
\widehat{H}=\widehat{H}_{0}-\widehat{V}_{12}-\widehat{V}_{13}-\widehat{V}_{23},
$$

где $\widehat{V}_{i j}$ - оператор умножения в $\ell_{2}\left(\left(\mathbb{Z}^{3}\right)^{3}\right)$ :

$$
\left(\widehat{V}_{i j} \hat{\psi}\right)\left(x_{1}, x_{2}, x_{3}\right)=\hat{v}_{i j}\left(x_{i}-x_{j}\right) \hat{\psi}\left(x_{1}, x_{2}, x_{3}\right), \quad \hat{\psi} \in \ell_{2}\left(\left(\mathbb{Z}^{3}\right)^{3}\right), \quad 1 \leqslant i<j \leqslant 3,
$$

$\hat{v}_{i j}$ - ограниченная вещественнозначная функция.

В дальнейшем предположим, что потенциал $\hat{v}_{i j}(s), 1 \leqslant i<j \leqslant 3$, является неотрицательной четной функцией на $\mathbb{Z}^{3}$ и удовлетворяет условию $\lim _{|s| \rightarrow \infty}|s|^{\rho} \hat{v}_{i j}(s)=0$. Здесь $\rho>3, s \in \mathbb{Z}^{3},|s|=\left|s^{(1)}\right|+\left|s^{(2)}\right|+\left|s^{(3)}\right|$.

Пусть $T^{3}$ - трехмерный тор, $L_{2}\left(\left(T^{3}\right)^{n}\right), n=1,2,3,-$ гильбертово пространство квадратично-интегрируемых функций, определенных на $\left(T^{3}\right)^{n}$.

Переход из координатного представления гамильтониана в импульсное осуществляется при помощи следующего преобразования Фурье:

$$
\mathcal{F}_{3}: L_{2}\left(\left(T^{3}\right)^{3}\right) \rightarrow \ell_{2}\left(\left(\mathbb{Z}^{3}\right)^{3}\right) .
$$


Обозначим через $\mathbf{H}=\mathcal{F}_{3}^{-1} \widehat{H} \mathcal{F}_{3}$ импульсное представление гамильтониана, описывающего систему трех произвольных квантовых частиц. Оператор Н действует в $L_{2}\left(\left(T^{3}\right)^{3}\right)$ по формуле

$$
\mathbf{H}=\mathbf{H}_{0}-\mathbf{V}, \quad \mathbf{V}=\mathbf{V}_{1}+\mathbf{V}_{2}+\mathbf{V}_{3} .
$$

Здесь

$$
\mathbf{H}_{0}=\frac{1}{2 m_{1}} \widehat{\Delta}_{k_{1}}+\frac{1}{2 m_{2}} \widehat{\Delta}_{k_{2}}+\frac{1}{2 m_{3}} \widehat{\Delta}_{k_{3}}
$$

и $\mathbf{V}_{i}, i=1,2,3,-$ интегральный оператор типа свертки

$$
\begin{aligned}
\left(\mathbf{V}_{\alpha} f\right)\left(k_{1}, k_{2}, k_{3}\right)= & \frac{1}{(2 \pi)^{3 / 2}} \int_{\left(T^{3}\right)^{3}} v_{\alpha}\left(k_{\beta}-k_{\beta}^{\prime}\right) \delta\left(k_{\alpha}-k_{\alpha}^{\prime}\right) \times \\
& \quad \times \delta\left(k_{\beta}+k_{\gamma}-k_{\beta}^{\prime}-k_{\gamma}^{\prime}\right) f\left(k_{1}^{\prime}, k_{2}^{\prime}, k_{3}^{\prime}\right) d k_{1}^{\prime} d k_{2}^{\prime} d k_{3}^{\prime},
\end{aligned}
$$

где

$$
v_{\alpha}(k)=\left(\mathcal{F}_{1}^{-1} \hat{v}_{\beta \gamma}\right)(k)=\frac{1}{(2 \pi)^{3 / 2}} \sum_{s \in \mathbb{Z}^{3}} \hat{v}_{\beta \gamma}(s) e^{i(k, s)}, \quad \alpha \neq \beta \neq \gamma,
$$

$\widehat{\Delta}_{k_{1}}=\widehat{\Delta} \otimes I \otimes I, \widehat{\Delta}_{k_{2}}=I \otimes \widehat{\Delta} \otimes I$ и $\widehat{\Delta}_{k_{3}}=I \otimes I \otimes \widehat{\Delta}, \widehat{\Delta}-$ оператор умножения на функцию $\varepsilon(k)$ :

$$
(\widehat{\Delta} f)(k)=\varepsilon(k) f(k), \quad \varepsilon(k)=\sum_{i=1}^{3}\left(1-\cos k^{(i)}\right) .
$$

Пусть $K=k_{1}+k_{2}+k_{3}$ - полный квазиимпульс системы трех частиц. Шестимерное многообразие $F_{K}=\left\{\left(k_{1}, k_{2}, k_{3}\right) \in\left(T^{3}\right)^{3}: k_{1}+k_{2}+k_{3}=K\right\}$ изоморфно тору $\left(T^{3}\right)^{2}$. Изоморфизм осуществляется с помощью отображения

$$
\Sigma_{K}: F_{K} \rightarrow\left(T^{3}\right)^{2}, \quad \Sigma_{K}((p, q, K-p-q))=(p, q) .
$$

Обозначим через $\mathbf{U}_{s}, s \in \mathbb{Z}^{3}$, оператор, действующий в $L_{2}\left(\left(T^{3}\right)^{3}\right)$ по формуле

$$
\left(\mathbf{U}_{s} f\right)\left(k_{1}, k_{2}, k_{3}\right)=e^{-i(s, K)} f\left(k_{1}, k_{2}, k_{3}\right) .
$$

Из-за того что оператор $\mathbf{H}$ коммутирует с унитарной группой $\left\{\mathbf{U}_{s}, s \in \mathbb{Z}^{3}\right\}, \mathbf{H}$ представляется в виде следующего прямого интеграла [5]:

$$
\mathbf{H}=\int_{T^{3}} \oplus \widetilde{H}(K) d K
$$

где ограниченный самосопряженный оператор $\widetilde{H}(K)$, действующий в $L_{2}\left(F_{K}\right)$, унитарно-эквивалентен оператору $H(K)$, действующему в $L_{2}\left(\left(T^{3}\right)^{2}\right)$ по формуле

$$
H(K)=H_{0}(K)-V, \quad V=V_{1}+V_{2}+V_{3} .
$$

Здесь $H_{0}(K)$ является оператором умножения на функцию

$$
\mathcal{E}_{K}(p, q)=\frac{1}{m_{1}} \varepsilon(p)+\frac{1}{m_{2}} \varepsilon(q)+\frac{1}{m_{3}} \varepsilon(K-p-q)
$$


и

$$
\begin{aligned}
& \left(V_{1} f\right)(p, q)=\frac{1}{(2 \pi)^{3 / 2}} \int_{T^{3}} v_{1}(q-s) f(p, s) d s \\
& \left(V_{2} f\right)(p, q)=\frac{1}{(2 \pi)^{3 / 2}} \int_{T^{3}} v_{2}(p-s) f(s, q) d s, \\
& \left(V_{3} f\right)(p, q)=\frac{1}{(2 \pi)^{3 / 2}} \int_{T^{3}} v_{3}(p-s) f(s, p+q-s) d s .
\end{aligned}
$$

Эквивалентность задается с помощью унитарного оператора $\widetilde{U}_{K}: L_{2}\left(F_{K}\right) \rightarrow$ $L_{2}\left(\left(T^{3}\right)^{2}\right)$, определенного по формуле $\widetilde{U}_{K} f=f \circ\left(\Sigma_{K}\right)$.

Введем оператор Шредингера $h_{\alpha}(k), k \in T^{3}$, соответствующий двухчастичному гамильтониану на решетке [6], действующий в $L_{2}\left(T^{3}\right)$ по формуле

$$
h_{\alpha}(k)=h_{0}^{\alpha}(k)-v_{\alpha},
$$

где $h_{0}^{\alpha}(k)$ - оператор умножения на функцию $\varepsilon_{\alpha}(k ; p)=\left(1 / m_{\beta}\right) \varepsilon(p)+\left(1 / m_{\gamma}\right) \varepsilon(k-p)$ и

$$
\left(v_{\alpha} f\right)(p)=\frac{1}{(2 \pi)^{3 / 2}} \int_{T^{3}} v_{\alpha}(p-s) f(s) d s .
$$

Обозначим через $v_{\alpha}^{1 / 2}(\cdot)$ ядро оператора $v_{\alpha}^{1 / 2}$, где $v_{\alpha}^{1 / 2}-$ положительный квадратный корень оператора $v_{\alpha}$.

ОПРЕДЕЛЕНИЕ. Говорят, что оператор $h_{\alpha}(0)$ имеет резонанс в нуле, если уравнение

$$
G_{\alpha}(0,0) \varphi=\varphi
$$

имеет единственное нетривиальное решение $\psi_{\alpha} \in L_{2}\left(T^{3}\right)$, удовлетворяющее условию $\int v_{\alpha}^{1 / 2}(q) \psi_{\alpha}(q) d q \neq 0$, где $G_{\alpha}(k, z)$ - интегральный оператор в $L_{2}\left(T^{3}\right)$ с ядром

$$
G_{\alpha}\left(k, z ; p, p^{\prime}\right)=\frac{1}{(2 \pi)^{3}} \int_{T^{3}} \frac{v_{\alpha}^{1 / 2}(p-t) v_{\alpha}^{1 / 2}\left(t-p^{\prime}\right)}{\varepsilon_{\alpha}(k ; t)-z} d t, \quad \alpha=1,2,3, \quad z \leqslant 0 .
$$

Заметим, что если нуль является резонансом оператора $h_{\alpha}(0)$ и $f_{\alpha}$ - соответствующее решение уравнения $h_{\alpha}(0) f=0$, то

$$
f_{\alpha}(p)=\frac{\left(v_{\alpha}^{1 / 2} \psi_{\alpha}\right)(p)}{\varepsilon_{\alpha}(0 ; p)} \in L_{1}\left(T^{3}\right) \backslash L_{2}\left(T^{3}\right),
$$

т.е. $z=0$ не является собственным значением оператора $h_{\alpha}(0)$.

Если уравнение (1) не имеет решения, то $\lambda=0$ не является собственным значением оператора $h_{\alpha}(0)$. В таком случае нуль называется регулярной точкой оператора $h_{\alpha}(0)$.

Можно проверить [7], что для всех $K \in T^{3}$ существенный спектр $\sigma_{\mathrm{ess}}(H(K))$ оператора $H(K)$ состоит из объединения спектров операторов каналов, т.е.

$$
\sigma_{\text {ess }}(H(K))=\sigma\left(H_{1}(K)\right) \cup \sigma\left(H_{2}(K)\right) \cup \sigma\left(H_{3}(K)\right), \quad H_{\alpha}(K)=H_{0}(K)-V_{\alpha} .
$$

Кроме того, если $H_{i}(0) \geqslant 0, i=1,2,3$, то $\sigma\left(H_{i}(0)\right)=\sigma\left(H_{0}(0)\right)=\left[0, M_{0}\right], M_{0}=$ $\max _{p, q} \mathcal{E}_{0}(p, q)$.

Теперь сформулируем основной результат данной работы. 
Теорема. Пусть нуль есть регулярная точка оператора $h_{\alpha}(0), H_{i}(0) \geqslant 0 u$ $V_{i} \geqslant 0, i=1,2,3$. Предположим также, что дискретный спектр $\sigma_{\mathrm{discr}}\left(H_{\beta \gamma}(0)\right)$ оператора $H_{\beta \gamma}(0)=H_{0}(0)-V_{\beta}-V_{\gamma}$ конечен и компактный оператор $v_{\alpha}$ является конечномерным, m.e.

$$
\operatorname{dim} \operatorname{Ran}\left(v_{\alpha}\right)<\infty
$$

Тогда оператор $H(0)$ может иметь лишь конечное число собственных значений, лежащих левее существенного спектра.

СЛЕДСТВИЕ. Пусть нуль есть регулярная точка оператора $h_{\alpha}(0)$ u onepamopы $h_{\beta}(0), h_{\gamma}(0)$ имеют резонанс в нуле. Предположим, что $H_{i}(0) \geqslant 0, V_{i} \geqslant 0$, $i=1,2,3, u m_{\alpha}=\infty, m_{\beta}<\infty, m_{\gamma}<\infty$. Если оператор $v_{\alpha}$ удовлетворяет условию (2), то дискретный спектр оператора $H(0)$ конечен.

ЗАмечАниЕ. Это следствие доказывает сделанное в работе [4] предположение для решетчатого случая для класса потенциалов $v_{\alpha}$, удовлетворяющих условию (2). При этом в случае выполнения условий следствия дискретный спектр соответствующего оператора Шредингера $H(0)$ конечен, если в трехчастичной системе частицы взаимодействуют с помощью парных контактных потенциалов притяжения.

\section{3. ДОКАЗАТЕЛЬСТВО ОСНОВНЫХ РЕЗУЛЬТАТОВ}

Приведем сначала некоторые свойства двухчастичного оператора Шредингера.

ЛЕмма 1. Пусть $h_{3}(0) \geqslant 0$ и нуль является регулярной точкой оператора $h_{3}(0)$. Тогда $\sigma\left(h_{3}(k)\right) \subset(0, \infty)$ для всех $k \in T^{3} \backslash\{0\}$.

ДокАЗАтЕЛьство. Пусть $h_{3}(0)$ - положительный оператор, т.е. $h_{3}(0) \geqslant 0$. Оператор $h_{3}(k)$ запишем в виде

$$
h_{3}(k)=\frac{m_{2}}{m_{1}+m_{2}} h_{3}(0)+\frac{m_{1}}{m_{1}+m_{2}} U_{k} h_{3}(0) U_{k}^{-1}, \quad k \in T^{3} \backslash\{0\},
$$

где $U_{k}: L_{2}\left(T^{3}\right) \rightarrow L_{2}\left(T^{3}\right)$ - унитарный оператор, определенный формулой $\left(U_{k} f\right)(q)=$ $f(k+q), f \in L_{2}\left(T^{3}\right)$. Из выражения (3) и положительности $h_{3}(0)$ имеем

$$
\left(h_{3}(k) f, f\right)=\frac{m_{2}}{m_{1}+m_{2}}\left(h_{3}(0) f, f\right)+\frac{m_{1}}{m_{1}+m_{2}}\left(U_{k} h_{3}(0) U_{k}^{-1} f, f\right) \geqslant 0
$$

для каждого $f \in L_{2}\left(T^{3}\right)$.

Пусть нуль является регулярной точкой оператора $h_{3}(0)$. Тогда $h_{3}(0) f \neq 0$ при $f \neq 0$. Отсюда имеем $\left(h_{3}(0) f, f\right)=\left(\left[h_{3}(0)\right]^{1 / 2} f,\left[h_{3}(0)\right]^{1 / 2} f\right)>0$, и из выражения $(3)$ вытекает, что $\left(h_{3}(k) f, f\right)>0$ для всех $k \neq 0$ и $f \neq 0$. Отсюда и из $\inf \sigma_{\text {ess }}\left(h_{3}(k)\right)=$ $m_{k}>0$ получаем включение $\sigma\left(h_{3}(k)\right) \subset(0, \infty)$ для всех $k \in T^{3} \backslash\{0\}$. Лемма 1 доказана.

Для оператора $G_{3}(k, z)$ имеем представление $G_{3}(k, z)=\left[v_{3}\right]^{1 / 2} r_{0}(k, z)\left[v_{3}\right]^{1 / 2}$, где $r_{0}(k, z)$ - оператор умножения на функцию $\left(\varepsilon_{3}(k ; p)-z\right)^{-1}, k \in T^{3}, z \leqslant 0$.

ЛЕмма 2. Пусть $h_{3}(0) \geqslant 0$ и нуль является регулярной точкой оператора $h_{3}(0)$. Тогда $\left\|G_{3}(k, z)\right\|<1$ для всех $k \in T^{3} u z \leqslant 0$. 
ДокАЗАТЕЛЬСтво. Предположим обратное, т.е. будем считать, что выполняются условия леммы, но $\left\|G_{3}(k, z)\right\| \geqslant 1$ для некоторого $k \in T^{3}$ и $z \leqslant 0$. Тогда оператор $G_{3}(k, z)$ имеет собственное значение $\lambda \geqslant 1$, поскольку он является положительным и компактным. Так как для ограниченных операторов $A$ и $B$ каждое ненулевое собственное значение оператора $A B$ является собственным значением и оператора $B A$ с той же кратностью (см., например, монографию [8]), то собственное значение $\lambda \geqslant 1$ оператора $\left[v_{3}\right]^{1 / 2} r_{0}(k, z)\left[v_{3}\right]^{1 / 2}$ является собственным значением оператора $r_{0}(k, z) v_{3}$ с той же кратностью. Пусть

$$
r_{0}(k, z) v_{3} \psi=\lambda \psi, \quad \psi \in L_{2}\left(T^{3}\right) .
$$

Тогда

$$
v_{3} \psi=\lambda h_{0}^{3}(k) \psi-\lambda z \psi, \quad \psi \in L_{2}\left(T^{3}\right)
$$

Отсюда

$$
0=\lambda h_{3}(k) \psi+(\lambda-1) v_{3} \psi-\lambda z \psi, \quad \psi \in L_{2}\left(T^{3}\right)
$$

или же

$$
\lambda\left(h_{3}(k) \psi, \psi\right)=(1-\lambda)\left(v_{3} \psi, \psi\right)+\lambda z(\psi, \psi) \leqslant 0, \quad k \neq 0 .
$$

Но согласно лемме 1 при $f \neq 0$ имеет место неравенство $\left(h_{3}(k) f, f\right)>0$. Это противоречие доказывает лемму 2.

Из доказанной леммы следует неравенство

$$
\left\|r_{0}(k, z) v_{3}\right\|<1 \quad \text { при } \quad k \in T^{3}, \quad z \leqslant 0 .
$$

Лемма 3. Операторнозначная функиия $R_{0}(\cdot) V_{3}$, определенная на $(-\infty, 0)$ со значениями в $L_{2}\left(\left(T^{3}\right)^{2}\right)$, является непрерывной в смысле операторной топологии на $(-\infty, 0), u \lim _{z \rightarrow-0} R_{0}(z) V_{3}=R_{0}(0) V_{3}$, где $R_{0}(z), z \leqslant 0$, - оператор умножсения на функиию $\left(\mathcal{E}_{0}(p, q)-z\right)^{-1}$.

ДокАзАТЕЛЬСтво. Из непрерывности функции $v_{3}(\cdot)$ в $T^{3}$ и векторнозначной функции $f(z)=\left(\mathcal{E}_{0}(p, q)-z\right)^{-1}$ на $(-\infty, 0)$ получаем непрерывность операторнозначной функции $R_{0}(\cdot) V_{3}$ на $(-\infty, 0)$ в смысле операторной топологии.

Заметим, что $(0,0) \in\left(T^{3}\right)^{2}$ является единственной невырожденной точкой минимума для функции $\mathcal{E}_{0}(\cdot, \cdot)$, и поэтому существуют числа $C^{\prime}, C^{\prime \prime}>0$ такие, что

$$
C^{\prime}\left(p^{2}+q^{2}\right) \leqslant \mathcal{E}_{0}(p, q) \leqslant C^{\prime \prime}\left(p^{2}+q^{2}\right) \quad \text { при } \quad p, q \in T^{3} .
$$

Следовательно, $R_{0}(0) V_{3}$ является ограниченным оператором в $L_{2}\left(\left(T^{3}\right)^{2}\right)$.

Из равенства

$$
\begin{aligned}
& \left\|\left(R_{0}(z) V_{3}-R_{0}(0) V_{3}\right) f\right\|^{2}= \\
& \quad=\int_{\left(T^{3}\right)^{2}}\left|\frac{z}{\left(\mathcal{E}_{0}(p, q)-z\right) \mathcal{E}_{0}(p, q)} \int_{T^{3}} v_{3}(s-p) f(s, p+q-s) d s\right|^{2} d p d q
\end{aligned}
$$


получим следующее неравенство для нормы оператора $R_{0}(z) V_{3}-R_{0}(0) V_{3}$ :

$$
\left\|R_{0}(z) V_{3}-R_{0}(0) V_{3}\right\|^{2} \leqslant C \int_{\left(T^{3}\right)^{2}} \int_{T^{3}} \frac{z^{2}}{\left|\left(\mathcal{E}_{0}(p, q)-z\right) \mathcal{E}_{0}(p, q)\right|^{2}}\left|v_{3}(s-p)\right|^{2} d s d p d q .
$$

Согласно неравенству (5) имеем

$$
\left\|R_{0}(z) V_{3}-R_{0}(0) V_{3}\right\|^{2} \leqslant C \int_{\left(T^{3}\right)^{2}} \frac{z^{2}}{\left|\left(p^{2}+q^{2}-z\right)\left(p^{2}+q^{2}\right)\right|^{2}} d p d q \leqslant C|z|,
$$

где $C>0$ не зависит от $z$. Отсюда получим

$$
\left\|R_{0}(z) V_{3}-R_{0}(0) V_{3}\right\| \rightarrow 0 \quad \text { при } \quad z \rightarrow-0 .
$$

Лемма 3 доказана.

Имеет место представление

$$
U R_{0}(z) V_{3} U^{-1} f\left(p_{1}, p_{2}\right)=\left[I \otimes r_{0}\left(p_{1}, z-\frac{1}{m_{3}} \varepsilon\left(p_{1}\right)\right) v_{3}\right] f\left(p_{1}, p_{2}\right),
$$

где $U: L_{2}\left(\left(T^{3}\right)^{2}\right) \rightarrow L_{2}\left(\left(T^{3}\right)^{2}\right)$ - унитарный оператор, определенный по формуле $U f\left(p_{1}, p_{2}\right)=f\left(p_{1}, p_{2}-p_{1}\right)$. Учитывая это представление, из неравенства (4) получим

$$
\left\|R_{0}(z) V_{3}\right\|<1 \quad \text { при } \quad z \leqslant 0 .
$$

Отсюда и из леммы 3 следует, что справедлива следующая

Лемма 4. Пусть $h_{3}(0) \geqslant 0$ и нуль является регулярной точкой оператора $h_{3}(0)$. Тогда оператор $\left(I-R_{0}(z) V_{3}\right)^{-1}$ при $z<0$ существует и определен в $L_{2}\left(\left(T^{3}\right)^{2}\right)$. Кроме того, $\lim _{z \rightarrow-0}\left(I-R_{0}(z) V_{3}\right)^{-1}=\left(I-R_{0}(0) V_{3}\right)^{-1}$ в смысле операторной топологии.

ДоКАЗАТЕЛЬСТво теОРЕмЫ. Не умаляя общности, допустим, что $\alpha=3, \beta=1$, $\gamma=2$.

Предположим обратное, т.е. будем считать, что условия теоремы выполняются, а оператор $H(0)$ имеет бесконечное число отрицательных собственных значений.

Обозначим через $\varphi_{1}, \ldots, \varphi_{k}, \ldots$ - собственные (ортонормальные) функции, соответствующие собственным значениям $z_{1} \leqslant \cdots \leqslant z_{k} \leqslant \cdots<0$. Так как $H_{i}(0) \geqslant 0$, то $\sigma_{\text {ess }}(H(0))=\left[0, M_{0}\right]$. Поэтому $\lim _{k \rightarrow \infty} z_{k}=0$.

По определению $\varphi_{i}$ и $z_{i}$ имеет место равенство

$$
H(0) \varphi_{i}=z_{i} \varphi_{i}
$$

или

$$
\varphi_{i}=R_{0}\left(z_{i}\right)\left(V_{1}+V_{2}+V_{3}\right) \varphi_{i}
$$

Согласно лемме 4 последнее равенство можно записать в виде

$$
\varphi_{i}=\left[I-R_{0}\left(z_{i}\right) V_{3}\right]^{-1} R_{0}\left(z_{i}\right)\left(V_{1}+V_{2}\right) \varphi_{i}
$$


или же

$$
\varphi_{i}=Q\left(z_{i}\right) \varphi_{i}
$$

где $Q(z)=\left[I-R_{0}(z) V_{3}\right]^{-1} R_{0}(z)\left(V_{1}+V_{2}\right)$.

Положим $K(z)=V_{3}^{1 / 2} Q(z), z<0$. Из лемм 3 и 4 получаем, что:

1) $K(z)$ является компактным оператором при каждом $z<0$;

2) операторнозначная функция $K(\cdot)$ является непрерывной в смысле операторной топологии на $(-\infty, 0)$, и $\lim _{z \rightarrow-0} K(z)=K(0)$.

Отсюда и из равенства $\left(V_{3} \varphi_{k}, \varphi_{k}\right)=\left(K^{*}\left(z_{k}\right) K\left(z_{k}\right) \varphi_{k}, \varphi_{k}\right)$ с учетом ортонормальности системы $\left\{\varphi_{k}\right\}$ вытекает, что $\left(V_{3} \varphi_{k}, \varphi_{k}\right) \rightarrow 0$ при $k \rightarrow \infty$. Так как по условию теоремы дискретный спектр оператора $H_{12}(0)$ конечен, то согласно равенству $z_{k} \varphi_{k}=H_{12}(0) \varphi_{k}-V_{3} \varphi_{k}$ имеем $V_{3} \varphi_{k} \neq 0$ для некоторого $N_{0}>0$ и для всех $k \geqslant N_{0}$.

Следовательно, существует подпоследовательность $\left\{\varphi_{n_{k}}\right\}_{k=1}^{\infty}$, для которой

$$
\left(V_{3} \varphi_{n_{k}}, \varphi_{n_{k}}\right)=\left\|V_{3}^{1 / 2} \varphi_{n_{k}}\right\|^{2}>0, \quad\left\|K\left(z_{n_{k}}\right) \varphi_{n_{k}}\right\| \leqslant \frac{C}{k^{2}}, \quad k=1,2, \ldots
$$

Пусть $\mathcal{H}_{0}$ - пространство собственных функций, соответствующих отрицательным собственным значениям $z_{n_{1}}, z_{n_{2}}, \ldots$ оператора $H(0)$, и

$$
K=\sum_{i=1}^{\infty}\left(\cdot, \varphi_{n_{k}}\right) K\left(z_{n_{k}}\right) \varphi_{n_{k}} .
$$

Легко проверить, что $K$ является компактным оператором. Пусть $P$ - проектор на $\mathcal{H}_{0}$. Тогда по определению оператор $P H(0) P$ имеет бесконечное число собственных значений.

При этом

$$
\widetilde{N}(P H(0) P)=\infty,
$$

где $\tilde{N}(A)=\operatorname{dim} \operatorname{Im} E_{(-\infty, 0)}(A),\left\{E_{\Omega}(A)\right\}$ - спектральное семейство оператора $A$.

Учитывая равенство $U V_{3} U^{-1}=I \otimes v_{3}$, из теоремы о спектре тензорного произведения операторов получим $\sigma\left(V_{3}\right)=\sigma\left(v_{3}\right)$. Из выражения (2) следует, что существует такое $\varepsilon>0$, для которого $\sigma\left(V_{3}\right) \cap(0, \varepsilon)=\varnothing$ и

$$
\sigma\left(P V_{3} P\right) \cap(0, \varepsilon)=\varnothing .
$$

Из равенства

$$
\left(P V_{3} P f, f\right)=\left(V_{3} \sum_{i=k}^{\infty}\left(f, \varphi_{n_{k}}\right) \varphi_{n_{k}}, \sum_{i=1}^{\infty}\left(f, \varphi_{n_{i}}\right) \varphi_{n_{i}}\right)
$$

и формулы (7) для всех $f \in \mathcal{H}_{0}$ имеем

$$
\left(P V_{3} P f, f\right)=\left(V_{3} \sum_{i=1}^{\infty}\left(f, \varphi_{n_{k}}\right) Q\left(z_{k}\right) \varphi_{n_{k}}, \sum_{i=1}^{\infty}\left(f, \varphi_{n_{i}}\right) Q\left(z_{i}\right) \varphi_{n_{i}}\right)=\left(K^{*} K f, f\right) .
$$

Следовательно, согласно выражению (8) операторы $K^{*} K$ и $P V_{3} P$ являются конечномерными в $\mathcal{H}_{0}$. Пользуясь неравенством (см. $\S$ ХIII.15 в книге [5])

$$
\widetilde{N}(P H(0) P) \leqslant \widetilde{N}\left(P H_{12}(0) P\right)+\widetilde{N}\left(-P V_{3} P\right)
$$


и учитывая конечность $\widetilde{N}\left(P H_{12}(0) P\right)$ и $\widetilde{N}\left(-P V_{3} P\right)$, имеем $\widetilde{N}(P H(0) P)<\infty$.

Это противоречие доказывает теорему.

ДОКАЗАТЕЛЬСТВО СЛЕДСТВИЯ. Не ограничивая общности, предположим, что $m_{1}, m_{2}<\infty, m_{3}=\infty$.

В этом случае операторы $H_{1}(0), H_{2}(0)$ и $H_{12}(0)$ определяются равенствами

$$
\begin{gathered}
H_{1}(0)=h_{1}(0) \otimes I+I \otimes h_{0}^{2}(0), \quad H_{2}(0)=h_{0}^{1}(0) \otimes I+I \otimes h_{2}(0), \\
H_{12}=h_{1}(0) \otimes I+I \otimes h_{2}(0),
\end{gathered}
$$

где $I$ - единичный оператор в $L_{2}\left(T^{3}\right)$. Так как $H_{i}(0) \geqslant 0, i=2,3$, из равенств (9) получим, что $\sigma_{\text {discr }}\left(h_{i}(0)\right)=\varnothing$. Из представления $(9)$ и теоремы о спектре тензорного произведения операторов имеем $\sigma\left(H_{12}\right)=\left\{\lambda+\mu: \lambda \in \sigma\left(h_{1}(0)\right), \mu \in \sigma\left(h_{2}(0)\right)\right\}=$ $\left[0, M_{0}\right]$, т.е. $\sigma_{\text {discr }}\left(H_{12}(0)\right)=\varnothing$. Отсюда и из теоремы получаем доказательство следствия.

\section{Список литературы}

[1] В. Н. Ефимов, ЯФ, 12:5 (1970), 1080-1091.

[2] Д. Р. Яфаев, Матем. сб., 94(136):4(8) (1974), 567-593.

[3] A. V. Sobolev, Comm. Math. Phys., 156 (1993), 101-126.

[4] H. Tamura, J. Funct. Anal., 95 (1991), 433-459.

[5] М. Рид, Б. Саймон, Методы современной математической физики. Т. 4. Анализ операторов, Мир, М., 1982.

[6] М.Э. Муминов, ТМФ, 148:3 (2006), 428-443.

[7] С. Н. Лакаев, М. Э. Муминов, ТМФ, 135:3 (2003), 478-503.

[8] П. Халмош, Гилъбертово пространство в задачах, М., Мир, 1970. 